\title{
Experiência do paciente: caminhos percorridos e a percorrer na coprodução do cuidado centrado no paciente
}

\author{
Patient experience: paths covered and to be covered in patient-centered care
}

Experiencia del paciente: caminos recorridos y por recorrer en la coproducción de una atención dirigida al paciente

ORCID: https://orcid.org/0000-0002-3484-0740 Universidade Federal de Santa Catarina, Brasil E-mail: bruna.canever@ufsc.br Luiz Eduardo Weimer

ORCID: https://orcid.org/0000-0003-3676-2982 Universidade Federal de Santa Catarina, Brasil E-mail: luizwweimer@gmail.com

Francys Kassick

ORCID: https://orcid.org/0000-0002-3089-3597

Universidade Federal de Santa Catarina, Brasil E-mail: francyskassick@gmail.com

Francis Ghignatti da Costa

ORCID: https://orcid.org/0000-0002-9597-2069

Universidade Federal de Santa Catarina, Brasil E-mail: frangcosta12@gmail.com

Andrieli Daiane Zdanski De Souza ORCID: https://orcid.org/0000-0002-3235-0182 Universidade Federal do Rio Grande do Sul, Brasil E-mail: adzsouza@hcpa.edu.br

Mellina Da Silva Terres

ORCID: https://orcid.org/0000-0003-3923-3736

\begin{abstract}
Resumo
Objetivo: Identificar iniciativas que demonstram a coprodução do cuidado centrado no paciente durante a internação hospitalar. Método: Estudo qualitativo, exploratório-descritivo, com triangulação de múltiplas fontes de dados: análise documental, observação de 10 profissionais e 24 entrevistas com pacientes-famílias. A análise temática sustentou-se no conceito de coprodução. Resultados: Caminhos percorridos e a percorrer emergiram das percepções dos participantes acerca da coprodução do cuidado centrado no paciente, canalizados na qualidade e segurança assistencial, informações e orientações como subsídio para participar do tratamento, atenção aos direitos e deveres e atendimento das necessidades de saúde. Conclusões: Ainda que tenha se identificado potencial para a coprodução rumo ao cuidado centrado no paciente, há caminhos a percorrer, na qualificação do atendimento das necessidades demandadas pelos pacientes-famílias. Considera-se que a experiência do paciente no processo de atendimento hospitalar, quando orientado pelos pressupostos da segurança do paciente, conduz à coprodução do cuidado centrado no paciente e promove a superação de barreiras para o avanço de parcerias entre profissionais e pacientes-famílias.

Palavras-chave: Segurança do paciente; Participação do paciente; Qualidade da assistência à saúde; Assistência centrada no paciente; Enfermagem.
\end{abstract}

\section{Abstract}

Objective: To identify initiatives that demonstrate the co-production of patient-centered care during hospitalization. Method: Qualitative, exploratory-descriptive study, with triangulation of multiple data sources: document analysis, 
observation of 10 professionals and 24 interviews with patient-families. Thematic analysis followed the concept of coproduction. Results: Paths covered and to be covered emerged from the participants' perceptions about the coproduction of patient-centered care, channeled in quality and safety of care, information and guidance as a subsidy to participate in treatment, attention to rights and duties, and meeting health needs. Conclusions: Although the potential for co-production towards patient-centered care has been identified, there are paths to go, in the qualification of the care of the needs demanded by patients and families. It is considered that the patient's experience in the process of hospital care, when guided by the assumptions of patient safety, leads to the co-production of patient-centered care and promotes the overcoming of barriers to the advancement of partnerships between professionals and patientfamilies.

Keywords: Patient safety; Patient participation; Quality of health care; Patient-centered care; Nursing.

\section{Resumen}

Objetivo: Identificar iniciativas que demuestren la coproducción de la atención dirigida al paciente durante la hospitalización. Método: Estudio cualitativo, exploratorio-descriptivo, con triangulación de múltiples fuentes de datos: análisis documental, observación de 10 profesionales y 24 entrevistas con pacientes-familiares. El análisis temático siguió el concepto de coproducción. Resultados: Los caminos recorridos y por recorrer surgieron de las percepciones de los participantes sobre la coproducción de la atención dirigida al paciente, canalizados hacia la calidad y seguridad de la atención, la información y la orientación como subsidio a la participación en el tratamiento, atención a los derechos y deberes y atención a las necesidades de salud. Conclusiones: Si bien se ha identificado el potencial de coproducción hacia la atención dirigida al paciente, existen caminos por recorrer en la calificación de la atención de las necesidades demandadas por los pacientes-familiares. Se considera que la experiencia del paciente en el proceso de atención hospitalaria, guiada por los supuestos de seguridad del paciente, conduce a la coproducción de una atención dirigida al paciente y promueve la superación de barreras para el avance de las alianzas entre profesionales y pacientes-familias.

Palabras clave: Seguridad del paciente; Participación del paciente; Calidad de la atención de salud; Atención dirigida al paciente; Enfermería.

\section{Introdução}

A busca por estratégias que contribuam para o avanço da segurança do paciente e, consequentemente, para a redução de danos desnecessários associados aos cuidados em saúde, tem destacado a temática sobre a participação do paciente em sua segurança, sendo uma delas a coprodução em saúde (Batalden, 2018; Costa et al., 2020). Tal direcionamento, impelido aos prestadores de serviço há mais de uma década por meio do movimento em prol da segurança, mantém-se atualizado no âmbito mundial, reforçando o compromisso de considerar o paciente o principal agente de sua segurança (World Health Organization, 2021). Em nível nacional, busca-se promover uma mudança na cultura dos serviços de saúde, no que se refere à participação do cidadão nos processos de segurança assistencial (Ministério da Saúde, 2014).

Nesse sentido, encontra-se na coprodução uma possibilidade para ampliar essa participação, a qual foi inicialmente conceituada para a área de Marketing de Serviços (Parks et al., 1981). Nos serviços de saúde, tem sido explorada como uma estratégia para promover a participação dos usuários, cujo resultado reflete o esforço conjunto entre profissionais da saúde e usuários (Costa, Borchardt \& Pereira, 2018; Costa, et al., 2020).

Assim, a experiência do paciente passa a ser interesse dos serviços de saúde, pois os pacientes apresentam uma perspectiva que difere das apresentadas pelos profissionais (O’Hara et al., 2018; Brookes et al., 2019). Com isso, podem contribuir para a qualidade assistencial (Costa et al., 2020; Costa, et al., 2020). Pauta-se na concepção de experiência do paciente como uma rede complexa de percepções significativas, as quais são cooptadas por meio de interações entre usuários e prestadores de serviço, imersas em uma cultura organizacional em todo o continuum de cuidados (Wolf, et al., 2014).

Consta na literatura há várias décadas, a proposição de engajar o paciente na avaliação da qualidade em saúde (Donabedian, 1990). Ao considerar os diferentes conceitos disponíveis acerca da qualidade, demarca-se na condução desta pesquisa o direcionamento do Programa Nacional de Segurança do Paciente (PNSP) (Ministério da Saúde, 2014), o qual orienta a adoção dos pressupostos donabedianos (Donabedian, 1990) para avaliação da qualidade do cuidado em saúde, haja vista a sua sustentação ser considerada mundialmente (Institute of Medicine, 2001). 
Nesse sentido, pauta-se na dimensão da qualidade que versa sobre o cuidado centrado no paciente, pela congruência à aceitabilidade, a qual consiste em um dos sete pilares da qualidade (Donabedian, 1990). No cuidado centrado no paciente as decisões clínicas pautam-se em valores como respeito às preferências, necessidades e valores individuais do paciente (Institute of Medicine, 2001). A aceitabilidade refere-se ao grau de conformidade do cuidado às expectativas do paciente (Donabedian, 1990).

No modelo de cuidado centrado no paciente baseado na coprodução, os profissionais de saúde fornecem informações e incentivam a participação ativa do paciente no processo de cuidado (Park, 2019). Nesse contexto, a interação entre pacientes e profissionais são norteados por valores como a igualdade, responsabilidade, flexibilidade e negociação (Costa, Borchardt \& Pereira, 2018).

Apesar dos avanços alcançados nas últimas duas décadas para melhoria da qualidade e segurança assistencial, com ampliação da discussão acerca da participação do paciente na sua segurança (Costa et al., 2020), ainda é elevada a incidência de eventos adversos evitáveis (Bates \& Singh, 2018). Tem-se como agravante a identificação de barreiras culturais que dificultam a participação do paciente nos processos assistenciais (Liang et al., 2018), tanto por falta de preparo das equipes para trabalhar com pacientes, como por pacientes e familiares desconhecerem como podem se engajar (Pomey et al., 2018).

Tais considerações, mobilizaram a seguinte questão de pesquisa: o processo de atendimento hospitalar, quando orientado pelos pressupostos da segurança do paciente, conduz à coprodução do cuidado centrado no paciente? Tem-se como objetivo identificar iniciativas que demonstram a coprodução do cuidado centrado no paciente durante a internação hospitalar.

\section{Metodologia}

O presente estudo foi desenvolvido na perspectiva da abordagem qualitativa, permitindo aos pesquisadores a aproximação, exploração e descrição do tema de pesquisa à luz da convicção teórica de que o serviço, na área da saúde, resulta de um processo de coprodução (Parks et al., 1981). O estudo ocorreu em um hospital universitário público da região sul do Brasil. Destaca-se que a avaliação da qualidade é uma prática adotada há mais de duas décadas no cenário pesquisado, o que permite considerar que está incorporada à cultura organizacional.

Os critérios de inclusão foram: pacientes internados por seis dias ou mais, para oportunizar maior interação com profissionais no recebimento dos cuidados; condições clínicas que permitissem o deslocamento ao local da entrevista e; interesse em relatar a experiência com o cuidado hospitalar. Foram incluídos familiares que acompanharam grande parte do período de internação, para garantir que tenham vivenciado a experiência de acompanhante de paciente. $\mathrm{O}$ critério de exclusão vinculou-se a dificuldades na articulação da fala, observada no momento do convite pela pesquisadora. A seleção dos participantes deu-se a partir do censo diário de 12 unidades de internação clínicas e cirúrgicas, sendo escolhido o primeiro paciente do sexo feminino e o primeiro do masculino. Todos os convidados aceitaram integrar o estudo.

Os profissionais incluídos no estudo foram aqueles que compõem o quadro efetivo de trabalhadores e que estavam em atividade laboral nos turnos definidos para observação. Registra-se que dois profissionais manifestaram recusa ao convite de participação. Mediante a aplicação desses critérios participaram da pesquisa 22 pacientes e oito familiares adultos internados, além de 10 profissionais da saúde que prestaram cuidados à beira leito aos pacientes internados no momento da observação, quais sejam: três enfermeiros, seis técnicos de enfermagem e um terapeuta ocupacional.

Cabe esclarecer que pacientes e seus familiares constituíram um segmento de participantes, sendo denominados no presente estudo como paciente-família. A composição da amostra foi intencional, considerando-se o planejamento de entrevistar dois pacientes-famílias por unidade, totalizando 24 entrevistas.

A coleta de dados secundários foi realizada pela pesquisadora principal, com base nos documentos que orientavam as 
rotinas assistenciais, descritos na forma de políticas e planos assistenciais, contidos no repositório institucional no mês de outubro de 2018. Foram selecionados os documentos que continham a descrição explícita do envolvimento do paciente-família no cuidado.

A coleta de dados primários deu-se através de entrevistas e observação de campo em novembro de 2018. As entrevistas foram conduzidas pela pesquisadora principal. As observações, do tipo passivas, foram desenvolvidas por outra integrante do grupo de pesquisa, capacitada para o desenvolvimento da técnica de coleta.

No segmento paciente-família, ocorreram 16 entrevistas exclusivas com os pacientes, duas exclusivas com um familiar e, em seis oportunidades, simultaneamente com paciente-família, totalizando 24 entrevistas, com duração entre 30 e 40 minutos. A coleta foi realizada em sala privativa, na unidade de internação, de forma a assegurar a privacidade e sua condução foi orientada por um roteiro semiestruturado, com base na Técnica de Incidente Crítico (TIC), a qual possibilita cooptar e aprofundar percepções acerca do objeto de interesse (Flanagan, 1973).

As observações ocorreram no ambiente dos quartos dos pacientes, em 30 oportunidades de cuidados diretos em que estiveram envolvidos os profissionais citados anteriormente, totalizando 16 horas. A observação também se guiou por um roteiro contendo a descrição do local e atividades executadas, diálogos e comportamentos, bem como registro das impressões do observador.

Com a TIC o pesquisador busca compreender comportamentos, situações e consequências presentes na memória (Flanagan, 1973) do paciente-família, estimulando sua evocação a partir de uma assertiva disparadora: Pense como foi o atendimento em relação aos cuidados relacionados à sua segurança e ao seu envolvimento nesses cuidados. A partir da lembrança da situação pelo entrevistado, o pesquisador estimula o relato por meio de um roteiro de questões preconizado pela TIC, direcionando a memória para a descrição de quem estava envolvido na situação, seus comportamentos, inclusive de si mesmo, o motivo que gerou a lembrança de tal situação e o que poderia ser diferente.

As entrevistas foram gravadas e, posteriormente, transcritas. O material oriundo da transcrição literal das entrevistas e dos registros das observações foi organizado em arquivo eletrônico com auxílio do software NVivo 11, pois essa ferramenta facilita as etapas de análise quando se utiliza múltiplas fontes de dados. Destaca-se, no entanto, que as etapas da análise temática - pré-análise, exploração do material, tratamento dos resultados e interpretação - foram realizadas por busca manual dos trechos e núcleos de sentido relacionados ao objeto investigado, por meio de leituras e releituras do material que constituiu o corpus de análise. Assim, a análise temática conjunta permitiu a identificação dos temas e agrupamentos, com vistas a configuração da categoria foco deste manuscrito (Minayo, 2014).

A pesquisa foi aprovada pelo Comitê de Ética em Pesquisa do campo pesquisado, destacando que os termos de utilização de dados institucionais e de consentimento livre e esclarecido compuseram a documentação ética do estudo. Um sistema de codificação composto por letras e números arábicos foi usado para assegurar o anonimato dos participantes. As letras "P", "F" e "O" designaram, respectivamente, paciente, familiar e observação, sendo seguidas do número que expressa a sequência cronológica de obtenção da informação. O percurso metodológico seguiu os critérios indicados para pesquisa qualitativa, descritos no check list COREQ.

\section{Resultados}

Participaram do estudo 22 pacientes, oito familiares e 10 integrantes da equipe que atuam diretamente no cuidado ao paciente, neste caso, três enfermeiros, seis técnicos de enfermagem e um terapeuta ocupacional.

A idade dos pacientes variou entre 34 e 75 anos, com mediana de 57 anos. Quanto ao sexo, os critérios de seleção da amostra direcionaram o resultado encontrado, sendo metade do sexo masculino. O período de internação variou entre seis e 52 
dias, com mediana de 14 dias de hospitalização. O grau de escolaridade distribuiu-se entre ensino fundamental (n=9; 37,5\%) e médio $(n=9 ; 37,5 \%)$, ensino superior $(n=5 ; 21 \%)$ e analfabeto $(n=1 ; 4 \%)$. As causas de internação hospitalar foram diversas, relacionadas a comorbidades agudas e crônicas, de origem clínica ou cirúrgica. Apresenta-se no Quadro 1, uma síntese da categoria emergente descrita neste manuscrito.

Quadro 1. Síntese dos resultados da pesquisa. Porto Alegre, RS, Brasil, 2018.

\begin{tabular}{|c|c|}
\hline \multicolumn{2}{|c|}{ Caminhos percorridos e a percorrer na coprodução do cuidado centrado no paciente } \\
\hline Temas & Subtemas \\
\hline \multirow[t]{3}{*}{ Considerações sobre qualidade e segurança assistencial } & Ausência de percepções \\
\hline & Aspectos indiretos ao cuidado \\
\hline & Segurança do cuidado percebida na relação paciente/ profissional \\
\hline \multirow{4}{*}{$\begin{array}{l}\text { Informações e orientações como subsídio para participar do } \\
\text { tratamento }\end{array}$} & Orientações e informações como propulsores da coprodução \\
\hline & Quando as informações esclarecem as dúvidas \\
\hline & Quando as informações não atendem às expectativas \\
\hline & Encontros oportunos para informar e orientar \\
\hline \multirow[t]{4}{*}{ Atenção aos direitos e deveres dos pacientes } & Presença do familiar acompanhando a internação \\
\hline & Necessidades especiais dos pacientes \\
\hline & Prestação de cuidados em tempo oportuno \\
\hline & Participação no cuidado por meio de consentimento \\
\hline \multirow[t]{7}{*}{ Atendimento das necessidades de saúde } & Avaliação da dor \\
\hline & Percepções dos padrões técnicos assistenciais \\
\hline & Tempo oportuno ou tardio para o atendimento \\
\hline & Apoio para as necessidades especiais \\
\hline & Horário das refeições de acordo com as preferências \\
\hline & $\begin{array}{l}\text { Oportunidades para investigar as necessidades de saúde dos } \\
\text { pacientes }\end{array}$ \\
\hline & Postura solícita da equipe de enfermagem \\
\hline
\end{tabular}

Fonte: Autores.

\subsection{Considerações sobre qualidade e segurança assistencial}

$\mathrm{Na}$ análise dos documentos institucionais, consta no planejamento estratégico do campo estudado um objetivo que destaca a atenção de excelência centrada na pessoa, cuja diretriz reflete o modelo almejado de atividades assistenciais voltado para a segurança do paciente. No entanto, a análise das entrevistas apontou diferentes considerações sobre qualidade e segurança assistencial. Alguns participantes lembraram sobre algum aspecto da segurança, mas não em relação aos cuidados assistenciais diretos e, outros, manifestaram que a assertiva inicial da entrevista não gerou nenhuma lembrança, conforme os relatos a seguir:

Sobre segurança? Não, não falaram com a gente sobre isso (F6).

O mais significativo que eu acho é a entrada no hospital, que precisa identificação, crachá. [...] porque não é qualquer um que chega ali e pode entrar (F9).

Por outro lado, alguns pacientes relataram aspectos relacionados à segurança do cuidado, a partir do atendimento que receberam dos profissionais do setor de emergência:

[...] eu fui bem tratada lá embaixo [emergência] também, apesar que é muita gente, mas a gente sabe que está sendo cuidado, as enfermeiras são umas heroínas (P1).

[...] a enfermagem é muito atenciosa e apesar da demanda, do caos, porque é muita gente, conseguiram se adaptar e são muito organizadas. [...] sempre conferem o nome direitinho [referindo-se à administração de medicamentos] (P5). 


\subsection{Informações e orientações como subsídio para participar do tratamento}

O diálogo estimulou lembranças aos participantes sobre profissionais que prestaram atendimento e como perceberam o seu envolvimento nos cuidados. Nesse sentido, as experiências destacaram momentos de apreensão das orientações acerca dos cuidados e das informações que receberam como propulsores da coprodução nos cuidados. Alguns exemplos destacaram sua participação no processo de identificação, de prevenção de quedas, de higienização das mãos, processo de consentimento e cirurgia segura. Com a análise documental, identificou-se que a descrição dos protocolos assistenciais mencionados pelos pacientes orienta formas de interação entre profissionais e pacientes.

Algumas situações são ilustradas nas falas a seguir:

Depois da cirurgia do coração eu assisti palestra aqui no hospital [...]. Assim eu aprendi como eu precisava me cuidar, o que eu precisava fazer (Pl).

[...] o médico falou comigo sobre isso, qual é minha parte no meu tratamento [...] (P2).

[...] eu vou fazer cirurgia no joelho amanhã, me explicaram todos os detalhes, eu tenho ciência do que eu tenho que fazer depois, quais cuidados, qual é a minha parte [...] (P8).

Por outro lado, quando as informações não suprem as expectativas de esclarecimentos, tornam-se também marcantes nas experiências dos pacientes:

O que me frustrou um pouco, foi o período que eu fiquei na emergência, nenhum médico ou enfermeira me diziam nada! Aí a gente fica sem saber o que vai acontecer (Pl).

[...] o que faltou foi explicar os porquês, os médicos precisam dizer as coisas, parece que a gente nunca sabe o que está acontecendo (F4).

[...] precisaria ter um pouco mais de atenção nas explicações [...] (P16).

Apesar das manifestações dos participantes registrarem insuficiência de informações, a partir das observações dos cuidados à beira-leito foi possível constatar que enfermeiros e técnicos de enfermagem orientam e esclarecem sobre os cuidados, explorando possíveis dúvidas, demarcando espaços oportunos para informar e orientar pacientes-famílias. Verificouse esse comportamento em algumas situações envolvendo administração de medicamentos, em que o profissional explicava o que estava administrando e sua indicação, esclarecendo dúvidas sobre os receios que alguns medicamentos causam no paciente-família $(\mathrm{O} 2, \mathrm{O} 3, \mathrm{O} 4, \mathrm{O} 12, \mathrm{O} 13, \mathrm{O} 17, \mathrm{O} 18$ e O22).

Também, se verificou em outras situações relacionadas à avaliação das condições gerais dos pacientes, que houve reforço de orientações específicas sobre o tratamento e sobre segurança (O25, O26, O27, O28, O29 e O30). Por exemplo, durante a avaliação de um paciente, o profissional questiona ao mesmo se sabe para que serve a pulseira amarela. Diante da negativa do paciente, explica sobre o risco de queda e sobre os cuidados para reduzir esse risco (O26).

A mesma postura de instigar dúvidas para promover o cuidado seguro com participação da família foi percebida na observação de um paciente recém internado no hospital $(\mathrm{O} 21)$. O técnico de enfermagem, durante o banho de leito, interage com o paciente-família, perguntando sobre o que sabem sobre medidas de precaução para portador de germes multirresistentes. Observou-se nesta situação (O21), além do diálogo, a demonstração de como usar os materiais, bem como para manusear os comandos da cama para prevenção de quedas e, em outra situação, a demonstração da higienização das mãos com álcool gel (O23). Nesse sentido, verificou-se que tais ações vão ao encontro das orientações institucionais acerca do papel de todos os profissionais na promoção da educação em saúde. 


\subsection{Atenção aos direitos e deveres dos pacientes}

Houve manifestações que destacaram a atenção aos direitos e deveres do paciente-família. Nesse sentido, verificou-se na análise dos documentos institucionais que há preocupação com tais aspectos do cuidado centrado no paciente, mediante a definição de política e procedimentos institucionais específicos. Citam-se como exemplos, a presença do familiar na internação, atenção para as necessidades especiais, prestação de cuidados no tempo oportuno e participação no cuidado por meio de consentimento. Nos registros seguintes, destaca-se como esses temas emergiram nas falas dos participantes:

[...] na noite passada eu dormi muito bem, porque quando me acordava e via minha filha aqui por perto, já sabia onde estava, quando estou sozinha não sei onde estou (P22).

[...] o que eu acho um diferencial aqui é o jeito que atendem as necessidades especiais dela [filha], aqui é bem diferenciado em relação a outras experiências que tivemos (F9).

[...] o que é muito bom é que não fazem nada sem a gente permitir. [...] hoje eu não aceitei fazer [o cuidado], eu pedi antes a avaliação do médico, eu me senti bem com isso (P17).

Essa temática também esteve presente nas observações, nos momentos dos cuidados, em várias situações pode-se constatar seu oferecimento, a fim de buscar o consentimento do paciente-família. Os cuidados compreenderam apoio para o banho (O23), higiene com troca de fralda $(\mathrm{O} 16)$ e administração de medicamentos $(\mathrm{O} 3, \mathrm{O} 17, \mathrm{O} 18, \mathrm{O} 22)$.

\subsection{Atendimento das necessidades de saúde}

O cuidado centrado no paciente é oportunizado e percebido pelos pacientes-famílias, nos momentos de atendimento das necessidades de saúde. Os relatos a seguir fazem referência à avaliação da dor, a qual consta nas políticas e planos institucionais do campo estudado:

[...] quando troca o turno, o enfermeiro já vem no quarto para avaliar como estou, perguntam se eu tenho dor, isso já é rotina, se antecipam (P12).

[...] o que fazem é sempre perguntar sobre isso [dor], não é para achar estranho, é para evitar que se tenha (P15).

As manifestações a seguir exemplificam como os padrões técnicos assistenciais atendem às necessidades de saúde dos participantes e que os padrões assistenciais institucionais estão incorporados nos comportamentos dos profissionais:

[...] o meu ponto chave foi quando eu fui para a cirurgia, a competência dos médicos foi um espetáculo, [...] aí dá para ver quem deixa a gente confiante (P21).

[...] eu passei por várias unidades [...] e o que eu notei que chama minha atenção é que todo mundo tem uma linha de pensamento muito parecida, pode-se dizer até idêntica, em relação às orientações. Dá para ver que o hospital se preocupa com isso (P23).

Outro tópico que se apresentou foi o tempo de espera para o atendimento do que se necessita, tanto no sentido de ocorrer de maneira oportuna, como no sentido de levar mais tempo do que o esperado:

[...] eu só consigo dormir se estou num lugar em silêncio, e teve uma noite que colocaram outro paciente comigo que ficou toda a noite agitado, gritando, eu fiquei muito mal, sabia que não era culpa dele. [...] mas aí eu conversei com a 
enfermeira e passou uns 20 minutos trocaram ele para outro quarto, que bom! (P19).

[...] o problema maior é quando tu chamas para alguma coisa que é considerada básica, aí demora, mas não é básica para quem está esperando, ficar esperando a troca da fralda [...] (F14).

O horário das refeições foi mencionado como marcante para o atendimento das necessidades, mas em sentido contraditório em relação às expectativas e preferências dos pacientes, conforme consta a seguir:

[...] nas outras internações meu marido sempre me ajudava, mas fiquei viúva e agora preciso de ajuda e as enfermeiras me ajudam sempre, [...] a cortar a carne, abrir as coisas que eu preciso, porque eu não consigo sozinha (P4).

[...] se pudesse mudar o padrão dos horários das refeiçães isso ia me ajudar. Porque já não tenho fome, aí eu tomo café e logo em seguida vem o almoço, os horários são muito perto um do outro e eu passei 30 anos tomando café às 8h e almoçando às $14 \mathrm{~h}$ [paciente em cuidado paliativo] (P15).

Durante as observações, foi possível acompanhar algumas avaliações do estado de saúde dos pacientes, oportunidade para a equipe investigar sobre necessidades de saúde. No entanto, constatou-se que em algumas avaliações o tempo despendido focava no motivo da internação, sem maior espaço de escuta do paciente. As situações observadas foram, nesses casos, de pacientes que já estavam internados há mais dias, condição que talvez explique esse comportamento pouco investigativo, considerando que os espaços de diálogo podem ter sido oportunizados nos dias iniciais de internação (O6, O8, O10, O14 e O15).

Em outra situação, envolvendo o atendimento a um paciente com internação recente, foi possível acompanhar orientações iniciais e o oferecimento dos serviços de apoio à família que acompanha pacientes com prognóstico reservado. $\mathrm{O}$ comportamento investigativo e acolhedor do profissional promove diálogo com o familiar com vistas a identificar necessidades de saúde do paciente e de apoio à família (O30).

As observações também revelaram o atendimento das necessidades de saúde demandadas pelos pacientes-famílias em diversas oportunidades, como avaliação da dor, posicionamento no leito, oferecimento de água, apoio para refeições, higiene, apoio para sentar-se na cadeira, para deslocar-se ao banheiro, atendimento de agitação e de sangramento. O que se destacou no comportamento de vários profissionais foi a postura solícita para o atendimento do que for necessário $(\mathrm{O} 3, \mathrm{O} 4, \mathrm{O} 7, \mathrm{O} 9, \mathrm{O} 11$, O14, O16, O17, O19, O21, O22, O23, O25, O26, O27, O28, O29 e O30).

\section{Discussão}

Com os resultados foi possível identificar caminhos percorridos e a percorrer na coprodução do cuidado centrado no paciente, segundo suas experiências. Para alguns pacientes, o assunto remeteu a aspectos indiretos da qualidade e segurança, como a circulação de pessoas no ambiente hospitalar ou a segurança patrimonial. Para os que internaram de forma não eletiva e que passaram pela experiência de cuidado no setor de emergência, caracterizado por constante superlotação, o tópico fez emergir percepções sobre condições estruturais de atendimento, devido ao desajuste entre demanda e capacidade de atendimento, gerando falta de espaço e de privacidade.

No entanto, tais condições, também alertaram os pacientes para alguns cuidados relacionados à segurança compondo suas experiências, suas memórias registradas. Esses cuidados referiram-se ao modo atencioso da equipe no atendimento dos pacientes, mesmo diante de tanta demanda, com percepções sobre a segurança na administração de medicamentos, com 
Research, Society and Development, v. 11, n. 1, e32911124899, 2022

(CC BY 4.0) | ISSN 2525-3409 | DOI: http://dx.doi.org/10.33448/rsd-v11i1.24899

conferência de sua identificação e o atendimento das necessidades em tempo oportuno. Tais resultados vão ao encontro de estudo que investigou acerca do conhecimento dos usuários de um hospital sobre segurança do paciente, no qual a maioria desconhecia o significado desse termo, no entanto que reconheciam a realização da conferência de sua identificação antes dos cuidados (Ramos et al., 2021).

Uma das barreiras identificadas para pacientes fornecerem feedback de suas experiências relacionadas à segurança, se refere à condição cognitivo-cultural acerca da temática (De Brún et al., 2016). Apesar de os pacientes considerarem que segurança é importante, o posicionamento dos participantes foi que segurança é uma condição padrão, que faz parte do contexto do cuidado daquele país. Tal sentido, embora implique em confiança na equipe e no sistema de saúde, sugere que a segurança não demanda maior atenção por parte dos pacientes.

Nos resultados de outro estudo, que investigou sobre como os pacientes compreendem e classificam questões de segurança (O'Hara et al., 2018), algumas percepções foram semelhantes ao resultado desta pesquisa, como privacidade e ambiente. Por outro lado, encontrou-se um sentido diverso ao desta pesquisa, pois a privacidade foi relacionada às informações de saúde do paciente e o ambiente a detalhes de conforto, como luminosidade ou barulho (De Brún et. al., 2016). Na presente pesquisa, o sentido para privacidade e ambiente ressaltou falta de elementos básicos para o atendimento, como macas, as quais, quando são disponibilizadas, encontram-se muito próximas umas às outras, incorrendo na falta de privacidade.

Uma pesquisa evidenciou a importância de os profissionais ouvirem o feedback dos pacientes, para promover mudanças nas práticas de saúde e melhorar a experiência do paciente (Gillis et al., 2017). Nesse sentido, as mudanças resultam da coprodução, a qual abarca relação de confiança entre pacientes e profissionais de saúde, além da educação do paciente, no sentido de subsidiá-lo de informações para participar do tratamento, cujas ações culminam com comprometimento, integração e tomada de decisão conjunta entre profissionais da saúde e pacientes (Costa, Borchardt \& Pereira, 2018).

O cuidado centrado no paciente emergiu das experiências dos participantes deste estudo, condição que implica em sua participação nas decisões em relação a sua saúde, em atenção aos direitos e deveres do paciente, bem como no atendimento das necessidades de saúde de modo individualizado, seguindo-se suas preferências e decisões. Tais resultados refletem o referencial de coprodução (Costa, Borchardt \& Pereira, 2018), no que tange a necessidade de o profissional de saúde avaliar o perfil de cada paciente, considerá-lo de forma integral e estabelecer uma comunicação clara e respeitosa com o paciente e seus familiares.

No entanto, os resultados do presente estudo demarcam caminhos percorridos, quando se alcança as expectativas dos pacientes-famílias e, caminhos a percorrer, quando consideram que as expectativas de esclarecimentos não foram supridas, ou quando os cuidados não ocorrem de modo oportuno. No modelo de cuidado centrado no paciente baseado na coprodução, os pacientes participam do processo de raciocínio clínico com os profissionais, o que propicia uma tomada de decisão informada, além do compartilhamento da sua história e preferências não clínicas (Park, 2019).

Na presente pesquisa, nos cuidados em que houve coprodução, como processo de identificação, prevenção de quedas, cirurgia segura, os pacientes-famílias se sentiram subsidiados para uma participação efetiva no tratamento, mediante a apreensão das informações e orientações recebidas. Ainda, em outras situações, pode-se constatar esse importante papel da equipe de saúde, para promover a participação dos usuários, como nos momentos de decisão sobre cirurgia, sobre mudança de algum comportamento necessário para o seguimento do tratamento, sobre ter conhecimento do seu plano de tratamento no continuиm de cuidados e, sobre o desenvolvimento de autonomia sobre sua saúde, considerando a continuidade do tratamento no pós-alta hospitalar. A postura da equipe de saúde, nesse sentido, em utilizar espaços oportunos para informar e orientar também foi constatada.

Estudos retratam a questão da comunicação e informações como importante fator para a segurança do paciente. Uma barreira que pode resultar na falta de interesse do paciente em participar da segurança inclui a falta de acesso a informações 
(De Brún et al., 2016; Marca-France et al., 2020). Destaca-se que a comunicação entre equipes e pacientes foi descrita como um dos principais fatores relacionados à segurança, na percepção de pacientes (O’Hara et al., 2018, Schenk et al., 2019). Essa condição aponta para valorização do preparo dos profissionais para a educação dos pacientes em relação à segurança assistencial. No entanto, consta na literatura algumas lacunas sobre como o paciente e família podem contribuir para a segurança do paciente, pela falta de espaço de escuta, além de como os profissionais podem promover a corresponsabilização da família no processo de cuidado (Schenk et al., 2019).

Para alguns participantes desta pesquisa, a falta de informações dificultou o acompanhamento do plano de tratamento, bem como algumas necessidades não foram atendidas em tempo oportuno. Com isso, nem todos se perceberam subsidiados com informações, em alguns casos houve manifestação de frustração em relação a isso. Consta na literatura que, ao experienciar situações que não correspondem às expectativas do paciente durante a hospitalização, há um impacto negativo nas suas percepções em relação ao hospital (Rapport et al., 2019). Por outro prisma, a capacitação da equipe para o cuidado centrado no paciente e o uso de tecnologias para envolver os pacientes foram associados a menores taxas de eventos adversos e maior satisfação (Dykes et al., 2017). Destaca-se que o uso de tecnologias de informação em saúde foi apontado como prioridade emergente para melhoria da segurança do paciente (Bates \& Singh, 2018).

O cuidado centrado no paciente abrange informações claras e compreensíveis, diálogo entre paciente e profissionais, demonstração de respeito, envolvimento nas decisões, privacidade, condições do ambiente, informações clínicas, tratamento e direitos, confiança nos profissionais, suporte emocional e autogestão do cuidado (Gomes \& Mendes Júnior, 2017).

Nesse sentido, no que tange aos caminhos percorridos, percebe-se elementos identificados neste estudo que propiciam a coprodução do paciente, uma vez que se verificou um processo de educação do paciente, assim como o desenvolvimento de ações que fortalecem a relação entre profissionais de saúde e pacientes, seguindo o disposto na literatura (Costa, Borchardt \& Pereira, 2018).

Em relação aos caminhos a percorrer, constataram-se algumas ações cuja coprodução é incipiente, tais como relacionadas à segurança ou ao atendimento de suas necessidades especiais. Pondera-se o grande desafio de um hospital universitário em ter processos relacionados à coprodução padronizados. Contudo, vale ressaltar que o desenvolvimento de protocolos que fomentem a coprodução, tais como descritos neste artigo, podem ser de grande valia uma vez que propiciam adesão e comprometimento ao tratamento, gerando melhores desfechos (Costa et al., 2018).

Considerou-se como limitação do estudo a coleta de dados primários ocorrer ao mesmo tempo do período de internação dos pacientes, condição que poderia gerar receio aos participantes de relatarem sobre seu atendimento, enquanto ainda o recebiam. Também, sabe-se que ao realizar observações sempre há o risco de mudanças nos comportamentos de quem está sendo observado, condição que caracteriza possível limitação. Apesar disso, o estudo possibilitou olhar para a temática da qualidade, nas dimensões segurança do paciente e cuidado centrado no paciente sob a ótica do receptor do serviço, ou seja, o paciente e a família, assumindo que são parte da coprodução. E, agregou, através da análise documental e observações de campo, o olhar para as definições institucionais e os comportamentos dos profissionais, pacientes e famílias nos momentos de interação, na prestação dos cuidados, ampliando a discussão sobre a temática no complexo cenário do atendimento em saúde.

\section{Considerações Finais}

Os achados demarcaram caminhos percorridos e a percorrer no cuidado centrado no paciente, apontando dessa forma, algumas lacunas que ainda se apresentam no ambiente de prestação de serviços, bem como avanços conquistados, os quais precisam ser disseminados e incorporados na cultura na medida em que forem apreendidos. Emergiram concepções sobre qualidade e segurança na percepção dos participantes, sendo fortemente marcadas por aspectos estruturais do atendimento, 
incorrendo na privacidade e no bem-estar dos pacientes. Houve constatação de oportunidades para coprodução, mediante o estímulo observado no comportamento de alguns profissionais, os quais provocaram pacientes-famílias a participarem de cuidados diretos à beira leito, com informações, orientações e esclarecimentos que subsidiaram o comportamento de coprodução.

Foi valorizada nas manifestações dos participantes, a atenção a seus direitos e deveres, mediante constante consentimento solicitado pelos profissionais para a execução do tratamento, além do atendimento de necessidades especiais e prestação de cuidados de acordo com as expectativas de entrega do serviço. Por outro lado, para alguns pacientes, a principal lacuna para a efetiva coprodução foram as informações insuficientes, que dificultaram o acompanhamento do tratamento e a falta de cuidados em alguns momentos demandados, gerando espera e sensação de julgamento sobre a importância do que foi solicitado.

Também, identificou-se no comportamento dos profissionais que não há constância em utilizar os momentos de interação como uma possibilidade de investigar as necessidades de saúde dos pacientes ou suas preferências, centrando o cuidado no paciente.

Considera-se que a experiência do paciente no processo de atendimento hospitalar, quando orientado pelos pressupostos da segurança do paciente, conduz à coprodução do cuidado centrado no paciente, condição que pode contribuir com a superação de barreiras culturais para o avanço de parcerias entre profissionais e pacientes-famílias.

O estudo busca contribuir para o avanço do conhecimento científico para a área de saúde e enfermagem com foco na participação do paciente no cuidado, teorizada no manuscrito com base na coprodução, relacionando-a com as dimensões da qualidade, quais sejam, segurança do paciente e cuidado centrado no paciente.

\section{Referências}

Batalden, P. (2018). Getting more health from healthcare: Quality improvement must acknowledge patient coproduction - An essay by Paul Batalden. BMJ (Online), 362. https://doi.org/10.1136/bmj.k3617

Bates, D. W., \& Singh, H. (2018). Two Decades Since To Err Is Human: An Assessment Of Progress And Emerging Priorities In Patient Safety. Health Affairs, 37(11), 1736-1743. https://doi.org/10.1377/hlthaff.2018.0738

Brookes, O., Brown, C., Tarrant, C., Archer, J., Buckley, D., Buckley, L. M., Clement, I., Evison, F., Gao Smith, F., Gibbins, C., Hayton, E., Jones, J., Lilford, R., Mullhi, R., Packer, G., Perkins, G., Shelton, J., Snelson, C., Sullivan, P., Vlaey, I., Wolstenholme, D., Wright, S. E. \& Bion, J. (2019). Patient experience and reflective learning (PEARL): a mixed methods protocol for staff insight development in acute and intensive care medicine in the UK. BMJ Open, 9(7), e030679. https://doi.org/10.1136/bmjopen-2019-030679

Costa, C. L. A., Borchardt, M. \& Pereira, G. M. (2018) O papel do paciente como coprodutor nos serviços de saúde: Uma revisão sistemática da literatura. Interciencia, 43(9), 648-654. https://www.interciencia.net/wp-content/uploads/2018/09/648-COSTA-43-09.pdf

Costa, D. G., Moura, G. M. S. S., Moraes, M. G., Santos, J. L. G. \& Magalhães, A. M. M. (2020). Satisfaction attributes related to safety and quality perceived in the experience of hospitalized patients. Rev Gaúcha Enf. 2020, 41(e20190152). doi: 10.1590/1983-1447.2020.20190152

Costa, D. G., Moura, G. M. S. S., Pasin, S. S., Costa, F. G. \& Magalhães, A. M. M. (2020). Patient experience in co-production of care: perceptions about patient safety protocols. Revista Latino-Am Enfermagem, 24(e.3272). doi: 10.1590/1518-8345.3352.3272

De Brún, A., Heavey, E., Waring, J., Dawson, P., \& Scott, J. (2016). PReSaFe: A model of barriers and facilitators to patients providing feedback on experiences of safety. Health Expectations, 20(4), 771-778. https://doi.org/10.1111/hex.12516

Donabedian, A. (1990) The seven pillars of quality. Arch of Pathol Lab Med., 114(11), 1115-1118. https://pubmed.ncbi.nlm.nih.gov/2241519/

Dykes, P. C., Rozenblum, R., Dalal, A., Massaro, A., Chang, F., Clements, M., Collins, S., Donze, J., Fagan, M., Gazarian, P., Hanna, J., Lehmann, L., Leone, K., Lipsitz, S., McNally, K., Morrison, C., Samal, L., Mlaver, E., Schnock, K., Stade, D., Yoon, C., Bates, D. W. (2017). Prospective Evaluation of a Multifaceted Intervention to Improve Outcomes in Intensive Care: The Promoting Respect and Ongoing Safety Through Patient Engagement Communication and Technology Study. Critical care medicine, 45(8), e806-e813. https://doi.org/10.1097/CCM.0000000000002449

Flanagan, J.C. (1973). A técnica do incidente crítico. Arq Bras Psicol, 25(2), 99- 141. http://bibliotecadigital.fgv.br/ojs/index.php/abpa/article/viewFile/16975/15786

Gillis, C., Gill, M., Marlett, N., Mackean, G., Germann, K., Gilmour, L., Nelson, G., Wasylak, T., Nguyen, S., Araujo, E., Zelinsky, S., \& Gramlich, L. (2017). Patients as partners in Enhanced Recovery after Surgery: A qualitative patient-led study. BMJ Open, 7(6). https://doi.org/10.1136/bmjopen-2017-017002 
Research, Society and Development, v. 11, n. 1, e32911124899, 2022

(CC BY 4.0) | ISSN 2525-3409 | DOI: http://dx.doi.org/10.33448/rsd-v11i1.24899

Gomes, P. H. G., \& Júnior, W. V. M. (2017). O cuidado centrado no paciente nos serviços de saúde: estratégias de governos e organizações não governamentais. Revista Acreditação: ACRED, 7(13), 23-43. https://dialnet.unirioja.es/servlet/articulo?codigo=6130783

Institute of Medicine (IOM) (2001). Committee on Quality of Health Care in America. Crossing the quality chasm: a new health system for the 21st century. Washington, DC: National Academy Press.

Liang, L., Cako, A., Urquhart, R., Straus, S. E., Wodchis, W. P., Baker, G. R., \& Gagliardi, A. R. (2018). Patient engagement in hospital health service planning and improvement: a scoping review. BMJ Open, 8(1), e018263. https://doi.org/10.1136/bmjopen-2017-018263

Marca-Frances, G., Frigola-Reig, J., Menéndez-Signorini, J. A., Compte-Pujol, M., \& Massana-Morera, E. (2020). Defining patient communication needs during hospitalization to improve patient experience and health literacy. BMC Health Services Research, 20(1). https://doi.org/10.1186/s12913-020-4991-3

Minayo, C. (2014). O desafio do conhecimento: pesquisa qualitativa em saúde. São Paulo Hucitec Rio De Janeiro Abrasco.

Ministério da Saúde (2014). Documento de referência para o Programa Nacional de Segurança do Paciente. Brasília: Ministério da Saúde. https://bvsms.saude.gov.br/bvs/publicacoes/documento_referencia_programa_nacional_seguranca.pdf

O’Hara, J. K., Reynolds, C., Moore, S., Armitage, G., Sheard, L., Marsh, C., Watt, I., Wright, J., \& Lawton, R. (2018). What can patients tell us about the quality and safety of hospital care? Findings from a UK multicentre survey study. BMJ Quality and Safety, 27(9), 673-682. https://doi.org/10.1136/bmjqs2017-006974

Park, S. (Ethan). (2019). Beyond patient-centred care: a conceptual framework of co-production mechanisms with vulnerable groups in health and social service settings. Public Management Review, 1-23. https://doi.org/10.1080/14719037.2019.1601241

Parks, R. B., Baker, P. C, Kiser, L., Oakerson, R., Ostrom, E., Ostrom, V., Percy, S. L., Vandivort, M. B., Whitaker, G. P. \& Rich Wilson. (1981). Consumers as co-producers of public services: some economic and institutional considerations. Policy Stud J., 9, 1001-11.

Pomey, M. P., Lebel, P., Clavel, N., Morin, É., Morin, M., Neault, C., Tétreault, B., \& Mulliez, A.-P. (2018). Development of Patient-Inclusive Teams: Toward a Structured Methodology. Healthcare Quarterly, 21(SP), 38-44. https://doi.org/10.12927/hcq.2018.25640

Ramos, R. S., Pina, R. M. P., Neves, J. C., Moura, G. S., \& Oliveira, H. M. (2021). O protagonismo de usuários em um hospital escola no Amazonas: Percepção acerca da segurança do paciente. Research, Society and Development, 10(3), e37510313597. https://doi.org/10.33448/rsd-v10i3.13597

Rapport, F., Hibbert, P., Baysari, M., Long, J. C., Seah, R., Zheng, W. Y., Jones, C., Preece, K., \& Braithwaite, J. (2019). What do patients really want? An indepth examination of patient experience in four Australian hospitals. BMC Health Services Research, 19(1). https://doi.org/10.1186/s12913-019-3881-z

Schenk, E. C., Bryant, R. A., Van Son, C. R., \& Odom-Maryon, T. (2019). Perspectives on Patient and Family Engagement With Reduction in Harm: The Forgotten Voice. Journal of nursing care quality, 34(1), 73-79. https://doi.org/10.1097/NCQ.0000000000000333

Wolf, J. A., Niederhauser, V., Marshburn, D., LaVela, S. L. (2014) Defining patient experience. Patient Exp J.,1(1), 7-19. http://pxjournal.org/journal/vol1/iss $1 / 3$

World Health Organization (2021). Strategic objective 4: Patient and family engagement. In: Global Patient Safety Action Plan 2021-2030. Genebra: World Health Organization. https://www.who.int/teams/integrated-health-services/patient-safety/policy/global-patient-safety-action-plan 